Cahiers d'études italiennes

\title{
Les nymphes de Boccace et l'essor du genre pastoral
}

\author{
Maria Cristina Panzera
}

\section{OpenEdition}

Journals

Édition électronique

URL : http://journals.openedition.org/cei/867

DOI : $10.4000 /$ cei.867

ISSN : 2260-779X

\section{Éditeur}

UGA Éditions/Université Grenoble Alpes

\section{Édition imprimée}

Date de publication : 15 juillet 2008

Pagination : 41-62

ISBN : 978-2-84310-122-9

ISSN : 1770-9571

Référence électronique

Maria Cristina Panzera, "Les nymphes de Boccace et l'essor du genre pastoral », Cahiers d'études italiennes [En ligne], 8 | 2008, mis en ligne le 15 janvier 2010, consulté le 19 avril 2019. URL : http:// journals.openedition.org/cei/867 ; DOI : 10.4000/cei.867 


\title{
LES NYMPHES DE BOCCACE \\ ET L'ESSOR DU GENRE PASTORAL
}

\author{
Cristina Panzera \\ Université Michel de Montaigne-Bordeaux 3
}

Le développement de l'imaginaire pastoral est un phénomène culturel marquant le renouveau des genres littéraires anciens à l'époque communale, sur une ligne de continuité bien connue qui relie l'œuvre de Dante à celle de Pétrarque et tout particulièrement à celle de Boccace ${ }^{1}$. Moins hanté que ses deux maîtres par le modèle virgilien, Boccace a su élargir cet imaginaire traditionnel qui se présentait à lui moins comme un genre littéraire défini, régi par des contraintes formelles et expressives rigoureusement établies - et une langue d'élection, le latin - que comme un réservoir très ouvert de motifs et de situations narratives, un décor à la fois stéréotypé et changeant où la représentation de la beauté féminine et de l'amour tiennent la première place, comme déjà chez Ovide ${ }^{2}$. En ce sens, le mot 'pastoral' assume dans l'œuvre de Boccace une connotation trans-générique, en amont de la ramification des genres qui permettra de distinguer par la suite, au cours de la Renaissance, une poésie, un roman ou un

1. Sur l'apport fondamental de Dante au développement de la poésie pastorale et sur ses continuateurs voir en particulier Giuseppe VELLI, "Tityrus redivivus': the Rebirth of Vergilian Pastoral from Dante to Sannazaro (and Tasso)", in Forma e parola. Studi in memoria di Fredi Chiappelli, éd. P. Mario Forni, Rome, Bulzoni, 1992, p. 67-79, mais également Domenico DE ROBERTIS, «L'egloga volgare come segno di contraddizione», in Metrica, II (1981), p. 61-80. Avec une perspective plus ample Elze KEGEL-BRINKGREVE, The echoing woods. Bucolic and pastoral from Theocritus to Wordsworth, Amsterdam, J.C. Gieben Publ., 1990, en particulier le chap. VI: «The Bucolic revival: italian eclogues 1320-1520», p. 273-284.

2. Pour l'apport de Boccace à la tradition pastorale voir en particulier Paolo Orvieto, " Boccaccio mediatore di generi e dell'allegoria d'amore», in Interpres, II (1978), p. 7-104, Gianvito RESTA, "Codice bucolico boccacciano", in I Classici nel Medioevo e nell'Umanesimo. Miscellanea filologica, Gênes, Istituto di filologia classica e medioevale, 1975, p. 59-90, Giuseppe VeLLI, « L'Ameto e la pastorale: il significato della forma", in ID., Petrarca e Boccaccio. Tradizione, Memoria, Scrittura, Padoue, Antenore, 1995, p. 195-208 et plus recemment Janet L. SMARR, "Boccaccio pastorale tra Dante e Petrarca", in Autori e lettori di Boccaccio, Atti del Convegno internazionale di Certaldo 20-22 settembre 2001, éd. M. Picone, Florence, Cesati, 2002, p. 237254. 
théâtre pastoral, car Boccace pastoral présente plusieurs visages, selon que l'on considère ses ouvrages écrits en langue vernaculaire ou en latin, en prose ou en vers, sur un arc temporel assez long qui va de la Chasse de Diane (vers 1334), c'est-à-dire des années du séjour napolitain de l'auteur, jusqu'à la fin des années soixante: 1367 est la date de composition présumée des dernières églogues du Buccolicum carmen ${ }^{3}$. Plus de trente ans de fidélité à une inspiration précoce - en 1334 Boccace avait à peine vingt ans - et tenace: dans le corpus pastoral de Boccace figurent certains passages du roman en prose Filocolo (1336-1338), la Comédie des nymphes florentines (ou Ninfale d'Ameto, vers 1341) qui reprend de la Vita Nuova la forme du prosimètre, c'est-à-dire une composition mixte de prose et de vers, et le Ninfale fiesolano (1344-1346) en huitains d'hendécasyllabes ${ }^{4}$.

Étant donné la variété de ce corpus, l'adoption d'un critère d'analyse trop sélectif risquerait d'engendrer des interprétations réductrices, comme dans l'importante monographie consacrée par Enrico Carrara à l'histoire de la poésie pastorale, monument de la critique positiviste italienne du siècle dernier, où Boccace apparait principalement comme l'auteur de la Comédie des nymphes florentines que le critique présentait, dans la lignée de la littérature didactique et allégorique médiévale, comme un simple développement du chant XXIX du Purgatoire de Dante ${ }^{5}$. En revanche, le caractère novateur et inventif de l'œuvre pastorale de Boccace a attiré plus récemment l'attention des critiques: aussi, son approche de la matière pastorale pourrait-elle être qualifiée de créative et expérimentale selon la remarque de Janet Smarr: "possiamo pensare che per Boccaccio la poesia pastorale non era una forma fissa né un soggetto fisso, ma anzi una possibilità di evocazione sempre flessibile; e renderci conto di come l'autore era sempre pronto ad esperimenti nuovi, che avrebbe continuato per tutta la vita $^{6} »$.

3. Giovanni Boccaccio, Buccolicum Carmen, éd. G. Bernardi Perini, in Tutte le opere di Giovanni Boccaccio, éd. Vittore Branca, vol. V, Milan, Mondadori, 1994.

4. Giovanni BocCACCIO, Caccia di Diana. Filocolo, in Tutte le opere..., cit., vol. I, 1967; ID., Comedia delle ninfe fiorentine, éd. A.E. Quaglio, Ibidem, vol. II, 1964; ID., Ninfale fiesolano, éd. A. Balduino, ibidem, 1974, vol. III. Dans nos citations du texte du Ninfale nous faisons référence au numéro des huitains.

5. Enrico CARRARA, La poesia pastorale, Milan, Francesco Vallardi, 1909, p. 156-161, en particulier p. 157 à propos de l'Ameto: «L'idillio è la visione del canto vigesimonono del Purgatorio, fu detto da altri, e possiamo aggiungere che, tolto il ciarpame mitologico ovidiano, in sostanza non è proprio altro che questo". L'étude de E. KeGEL-BRINKGREVE, The echoing woods..., cit., porte principalement sur la tradition de l'églogue classique; pour quelques remarques sur le Bucolicum carmen de Boccace, p. 273-284.

6. J. SMARR, «Boccaccio pastorale... », cit., p. 254: «Nous pouvons penser que pour Boccace la poésie pastorale n'était pas une forme figée, ni un sujet figé, mais au contraire une possibilité 
Une recherche sur l'héritage de Boccace pastoral à la Renaissance - qui reste fondamentalement à faire - pourra se fonder utilement sur ces notions de variété et d'expérimentalisme permettant de détacher l'œuvre de Boccace de la tradition pastorale de son temps, afin d'établir non seulement si ses différents ouvrages ont été lus et imités, et dans quelle mesure, mais aussi si les ferments nouveaux que cet auteur avait apportés à la tradition du genre ont trouvé des continuateurs, en élargissant les horizons et les fonctions de l'imaginaire pastoral. Plusieurs aspects sont à prendre en compte: peut-on affirmer, par exemple, que Boccace a légué à ses continuateurs les traits caractérisant son ouvre pastorale, à savoir la variété des solutions formelles, l'importance des éléments narratifs, la forte inspiration ovidienne, plus généralement la liberté par rapport aux schémas de la bucolique classique latine? Comment définir, en outre, les fonctions et les enjeux idéologiques de l'écriture pastorale chez les premiers imitateurs de Boccace? En effet, l'ouverture aux implications extra-littéraires de l'imaginaire pastoral représente une démarche nécessaire si l'on considère comment le mythe arcadien s'associe depuis ses origines à une réflexion d'ordre politique ou philosophique concernant par exemple le rôle du poète dans la société, son rapport au pouvoir, le rêve d'une paix à rétablir ou de l'harmonie perdue, l'âge d'or de Saturne'. Cependant, si ces aspects apparaissent centraux dans la recherche récente sur la littérature courtisane et le monde des cours, peu d'études s'étendent à l'époque antérieure, notamment pour l'œuvre de Boccace.

L'essor de la littérature pastorale s'accompagne du rayonnement culturel et politique de Florence et de la Toscane sous l'influence de Côme l'Ancien et de Laurent de Médicis, à partir de la moitié du XV siècle ${ }^{8}$. Force est de constater que Boccace avait particulièrement contribué à cet ancrage du mythe arcadien dans sa région natale: en effet, le parcours qui va de la Comédie des nymphes florentines au Ninfale Fiesolano semble confi-

d'évocation toujours flexible, comprenant ainsi comment l'auteur était toujours prêt à de nouvelles expérimentations qu'il aurait continuées tout au long de sa vie».

7. Voir en particulier pour tous ces aspects Annabell PATTERSON, Pastoral and Ideology. Virgil to Valéry, Berkeley, University of California Press, 1987.

8. Mario Martelli, Letteratura fiorentina del Quattrocento. Il filtro degli anni Sessanta, Florence, Le Lettere 1996, et ID., "La cultura letteraria nell'età di Lorenzo", in Lorenzo the Magnificent Culture and Politics, éd. Michael Mallet \& Nicholas Mann, Londres, The Warburg Institut, 1996, p. 167-176. En outre Paolo Orvieto, Pulci medievale. Studio sulla poesia volgare fiorentina del Quattrocento, Rome, Salerno Editrice, 1978, et Stefano CARRAI, Le muse dei Pulci. Studi su Luca e Luigi Pulci, Naples, Guida, 1985. 
gurer une spécialisation de l'imaginaire pastoral autour du mythe de Florence et de ses origines, selon une intention panégyrique renforçant l'identité et l'orgueil municipaux. Dans la Comédie des nymphes la rencontre d'Ameto avec sept nymphes d'une merveilleuse beauté symbolise son initiation aux vertus à une époque très ancienne avant la fondation de Fiesole, alors que le Ninfale fiesolano décrit le passage d'une préhistoire mythique des habitants de Fiesole - temps du péché symbolisé par l'union impie d'Africo et Mensola - au temps de l'histoire marqué par la fondation de la ville de Florence: «ma poi che Carlo Magno ebbe vittoria / passò di qua nelle nostre contrade / e rifece la città di Fiorenza / la qual poi crebbe ogni dì sua potenza ${ }^{\natural} »$. On comprend, alors, le lien identitaire très fort qui assure la continuité de ces ouvrages de Boccace à Florence. Il semblerait par exemple que le Ninfale Fiesolano ait profondément marqué la culture florentine, à en juger par ses nombreux échos signalés chez plusieurs auteurs du XV siècle, comme Laurent de Médicis, Ange Politien et les frères Pulci, mais aussi dans la littérature en langue latine puisque Mario Martelli en a donné des exemples pour la Flametta de l'humaniste Ugolino Verino $(1464)^{10}$. En reprenant les grandes lignes de cette opération culturelle axée sur la valorisation de la tradition florentine en langue vulgaire, nous allons nous intéresser à la place assignée à Boccace pastoral par les écrivains de l'époque humaniste et notamment par les frères Pulci, mais d'abord un détour par le théâtre et la peinture nous montrera l'étendue et l'importance de cet héritage.

À la fin de l'acte II de l'Aminta, la nymphe Sylvie s'apprête à entrer dans les eaux d'une source avant de se rendre au temple de Diane: va-t-elle succomber aux assauts amoureux du berger Aminta? Va-t-elle subir l'agression du grossier satyre qui vient aussitôt contrer les projets d'Aminta? Le Tasse entretient savamment le suspense en exploitant un motif devenu certes traditionnel, mais dont Boccace avait déjà fourni une version remarquable, sans doute la première, dans ce genre, au sein de la littérature italienne: la scène du viol de la nymphe Mensola qui constitue l'épisode central du Ninfale fiesolano ${ }^{11}$.

9. Ninf. Fies. 463: «Mais après sa victoire, Charlemagne passa par nos contrées et rebâtit la ville de Florence qui ensuite n'a cessé de redoubler toujours plus de puissance».

10. M. Martelli, Letteratura fiorentina, cit., p. 92. Pour les échos du Ninfale fiesolano chez Laurent de Médicis, cf. les notes de P. Orvieto à son édition du Canzoniere, Milan, Mondadori, 1984, notamment au sonnet 55, p. 127. Pour la présence du Ninfale dans les Stanze de Politien cfr. Lucia Battaglia RicCi, Boccaccio, Rome, Salerno Editrice, 2000, p. 119.

11. Le motif du viol n'est pas fréquent dans la littérature médiévale et apparaît principalement dans les fabliaux et dans les lais; voir Dietmar RIEGER, «Le motif du viol dans la littérature de la 
En remontant un peu dans le temps, nous retrouvons ce même épisode à la source d'un travail remarquable d'artisanat artistique en Toscane: un artiste de la première moitié du XV siècle a voulu représenter cette scène d'agression sur la partie antérieure d'un coffre en bois, un produit typique des ateliers toscans de l'époque, destiné souvent à être offert en cadeau de mariage. Reproduit dans le deuxième volume du beau recueil consacré à Boccaccio visualizzato avec trois autres coffres peints qui témoignent de la fortune iconographique du Ninfale, cet ouvrage est conservé au Bowdoin College Museum of Art de Brunswick, aux États-Unis, et il a été attribué par Fern Rusk Shapley au peintre Giovanni Toscani ${ }^{12}$. Plus récemment, à l'occasion d'une exposition au Musée Metropolitain de New-York, les spécialistes ont proposé une nouvelle attribution: d'après le catalogue, cette peinture serait l'œuvre de frère Jean, ou Fra Angelico, qui l'aurait réalisée en 1420, quand il était jeune moine dans le couvent de San Domenico à Fiesole ${ }^{13}$.

Dans le panneau antérieur de ce coffre, l'artiste a résumé, avec quelques infidélités au texte source, quatre moments de l'histoire du berger Africo: au début on le voit à peine à gauche, caché derrière un rocher en train de guetter le conseil de la déesse Diane et de ses nymphes qui ont toutes prononcé les vœux de chasteté. Amoureux de la nymphe Mensola, il désespère de pouvoir l'approcher, mais une nuit Vénus lui apparaît en rêve pour lui suggérer un plan : il devra se déguiser en nymphe pour se rapprocher de Mensola et entrer enfin avec elle dans la rivière où il pourra la violer. Pour ce passage, Boccace s'était vraisemblablement inspiré des Métamorphoses d'Ovide, notamment du mythe de la nymphe Callisto que

France médiévale: entre norme courtoise et réalité courtoise", in Cahiers de civilisation médiévale, 31 (1988), p. 241-267.

12. Boccaccio visualizzato. Narrare per parole e per immagini fra Medioevo e Rinascimento, éd. V. Branca, 3 vol., Turin, Einaudi 1999, vol. II: Opere d'arte d'origine italiana, Turin, Einaudi, 1999. Dans la section I dipinti Massimiliano Rossi illustre quatre ouvrages inspirés du Ninfale, le coffre du Bowdoin College est décrit au $n^{\circ} 61$. Au n ${ }^{\circ} 60$ figure un coffre attribué au peintre Giovanni Toscani (conservé à New York, M. Roy Fischer Fine Arts Inc.); au n 62 un panneau d'artiste florentin mis en vente par Christie's en 1985 et au no 63 un autre panneau de la première moitié du XV siècle, toujours d'artiste florentin, conservé au Musée Bonnat de Bayonne. Il existe également un certain nombre de manuscrits du Ninfale comportant des dessins et des scènes enluminées, voir dans Boccaccio visualizzato les fiches préparées par Maria Grazia CIARDI DUPRÉ DAL PogGetTo dans la section Codici miniati schede: les $\mathrm{n}^{\circ} 22,23$ et 40 montrent respectivement le ms. B. R. 47 (II. II. 66) de la Bibliothèque Nationale de Florence, de la première moitié du XV $\mathrm{XV}^{\mathrm{e}}$ le ms. It. IX. 63 de la Marciana de Venise et le ms. 1503 de la Bibliothèque Riccardiana de Florence, daté de 1482.

13. Laurence Kanter \& Pia Palladino, Fra Angelico, The Metropolitan Museum of Arts, New Haven-Londres, Yale University Press, 2005, p. 19-21. 
Zeus avait séduite prenant la figure et les habits de Diane (Métamorphoses II, 401-495). Dans la troisième scène du coffre, Africo prend alors congé de ses parents et part à la recherche de Mensola: la dernière scène nous le montre en train de saisir sa belle, tandis que les autres nymphes prennent la fuite ${ }^{14}$. Plusieurs mythes ovidiens se trouvent à l'origine de cet épisode du Ninfale, comme celui d'Actéon qui surprend Diane dans la source ou le récit de l'accouplement de la nymphe Salmacis et d'Hermaphrodite ${ }^{15}$.

La suite tragique de l'histoire du Ninfale n'est pas représentée dans le coffre, à savoir le suicide d'Africo abandonné par Mensola et la mort de la nymphe décrétée par Diane au moment où elle découvre qu'un enfant est né de cette relation interdite, situation qui rappelle encore le mythe ovidien de Callisto. Après leur mort, les deux amants subissent un sort commun, car ils donneront leur nom à deux rivières : pour Mensola, en particulier, il s'agit d'une métamorphose fluviale infligée par Diane, suivant un type de métamorphose qu'Ovide avait représentée à plusieurs reprises, par exemple pour la nymphe Aréthuse, pour Cyanée ou pour le berger Acis ${ }^{16}$. Africo, quant à lui, se donne la mort par désespoir près d'une rivière y déversant tout son sang. Cette double métamorphose fluviale rapproche le Ninfale fiesolano d'un genre caractéristique de la littérature alexandrine, le petit poème mythologique, en grec épillion, qui développe l'étiologie, c'est-à-dire les origines et les causes d'un élément ou d'un phénomène naturel.

De cette histoire si sombre et pathétique, l'artiste de notre coffre n'a privilégié donc que la première partie, de la naissance du désir à l'assouvissement de la passion sensuelle, un sujet parfaitement adapté à la destination nuptiale de l'objet et non dépourvu, sans doute, d'une finalité exemplaire. Il est difficile d'établir si le peintre avait une connaissance directe ou indirecte du Ninfale, mais l'histoire devait être suffisamment connue pour permettre aux commanditaires et à leur entourage de comprendre le message des scènes peintes. Cependant, l'artiste a fait preuve d'une certaine liberté d'interprétation, sorte de réécriture picturale, comme il apparaît dans les deux scènes centrales de la vision de Vénus et du départ d'Africo de sa famille. Dans le Ninfale Boccace avait raconté en ces termes l'apparition de la déesse et de Cupidon au jeune héros endormi:

14. Le décor pour la scène du viol dans la peinture paraitt très proche du texte source qui parle d'un lac entouré de montagnes: "Né furon guari le ninfe oltre andate, / che trovaron due ninfe tutte ignude / che 'n un pelago d'acqua erano entrate / dove l'un monte con l'altro si chiude", Ninf. Fies. 235.

15. Respectivement Ovide, Métam. III 139-252 et IV 271-415.

16. Respectivement Métam. V 572, V 409 et XIII 750. 
Pur alla fine, già press'al mattino

il sonno vinse gli occhi dell'amante:

e leggiermente dormendo supino,

Venere iddea gli venne davante,

e 'n collo avea Amor, picciol fantino,

con l'arco e le saette minacciante;

poi gli pareva che Venere iddea

cota' parole verso lui dicea ${ }^{17}$.

Mais dans la scène correspondante à ce passage, le peintre a représenté Vénus seule, sans le personnage de Cupidon, et nue, un détail absent du Ninfale mais en rapport sans doute avec le thème de la luxure implicite dans le récit. Autre liberté: sur le devant de la scène, en premier plan par rapport à Africo endormi dans son lit, une femme assise est en train de filer, la quenouille à la main. Il s'agit vraisemblablement d'Alimena, la mère d'Africo, car c'est dans la même robe grise qu'elle apparaît dans la séquence suivante, accompagnée de son mari Girafone. Ce détail narratif manque dans le récit de Boccace.

Une logique de l'amplification semble inspirer également la troisième scène du coffre. Dans le texte de Boccace, Africo, après la vision de Vénus, profite de l'absence de ses parents pour voler une robe à sa mère et le lendemain, de bon matin, il quitte la maison «alla madre dicendo - I'vo alla caccia». Le peintre a voulu mettre en relief ce moment de séparation en représentant à côté de la mère d'Africo le vieux père Girafone et sur la droite la silhouette élancée du jeune homme s'appuyant sur sa lance. Le langage gestuel des mains est très expressif et suffit à lui seul à donner l'ambiance de la scène: le doigt levé du père est un geste sévère d'avertissement et de mise en garde, alors que le fils, avançant en direction du vieil homme, la paume de sa main gauche ouverte, semble vouloir rassurer et accepter les conseils; moment symbolique d'affranchissement de l'autorité parentale qui prélude à la conclusion tragique représentée dans la dernière scène, celle de l'agression sexuelle.

De fortes ressemblances existent entre le coffre de Fra Angelico et un autre de la même époque attribué au peintre Giovanni Toscani ${ }^{18}$. La figure de la fileuse y apparaît également dans la scène de la vision de Vénus, mais la déesse se montre habillée et le personnage de Cupidon est

17. Ninf. Fies. 198: "Mais enfin peu avant la pointe du jour / le sommeil vainquit les yeux de l'amant / et à lui qui dormait allongé / se présenta la déesse Venus / portant dans ses bras son enfant, Amour, / qui menaçait de son arc et des flèches. / Il lui sembla ensuite que la déesse Vénus / lui adressait ce discours".

18. Boccaccio visualizzato, cit., $\mathrm{n}^{\circ} 60$. 
présent: il se tient debout comme une statuette sur la main droite de Vénus, alors que le texte le dit "n collo", à savoir dans les bras de sa mère. Ensuite, le groupe formé par Alimena, Girafone et Africo est représenté assis, toujours avec la même gestuelle des mains, sauf qu'Africo, le genou gauche à terre, tend la main droite en direction de son père et non la gauche. En revanche, Giovanni Toscani change complètement la dernière scène au profit d'une version plus épurée, car l'agression dans les eaux du lac n'est pas représentée et on voit seulement un groupe de trois nymphes derrière un puits en marbre de forme rectangulaire. Sans doute l'une des trois nymphes est à identifier avec Girafone qui, suivant le conseil de Vénus, s'est habillé en femme, peut être la figure à l'extrême gauche; d'ailleurs on reconnait à l'angle droit du panneau une robe de couleur foncée abandonnée à terre, comme dans le récit de Boccace lorsque Africo décide d'entrer nu dans la rivière où Mensola se baigne insouciante.

Malgré ces quelques libertés par rapport au Ninfale, le sujet des deux coffres devait être facilement lisible par le public: il est possible, par exemple, que les quatre scènes représentées symbolisent les différentes étapes de l'amour, de la rencontre avec l'être cher, la visio, à l'intériorisation de la passion à travers la pensée obsédante et le rêve, pour ensuite montrer le départ de l'amant de sa famille et la naissance d'une relation amoureuse dont l'union sexuelle représente le couronnement. Cette réception profane du Ninfale nous montre, en tout cas, que l'imaginaire pastoral de Boccace n'était pas limité au cercle restreint de la communication littéraire, il avait trouvé d'autres voies de diffusion et d'influence au niveau des pratiques sociales et artistiques. Mais un autre aspect mérite d'être considéré et concerne le langage pictural adopté par l'artiste afin de visualiser, traduire en images, ce que Boccace avait confié à l'art du récit.

La qualité artistique de la peinture dans le coffre de Brunswick et l'élégance des solutions chromatiques ont sûrement joué pour sa récente attribution à Fra Angelico. Mais au-delà de la question de l'attribution, il ne faudrait pas passer sous silence ce qui caractérise cet ouvrage encore plus que celui de Giovanni Toscani, à savoir la forte influence du modèle de Giotto, comme le révèlent certains détails concernant la représentation de l'espace et des personnages. Force est de constater, par exemple, la ressemblance entre le décor rocheux qui permet de séparer les quatre scènes et les rochers blancs des fresques de la Chapelle Scrovegni de Padoue. De même, la représentation des corps en transparence dans l'eau pourrait évoquer la scène du baptême du Christ à Padoue. Il faut remarquer, d'ailleurs, que ces deux détails, les roches et le lac, sont absents du tableau de Giovanni Toscani, où les groupes des personnages se suivent avec 
une impression d'entassement dans l'espace et où la scène du viol a été censurée.

Mais l'analogie la plus frappante avec les fresques de Giotto concerne le personnage au centre du tableau, la figure déjà mentionnée de la fileuse, absente du récit de Boccace, qui rappelle un détail de l'annonciation à saint'Anne de Giotto à Padoue: assise à l'extérieur de la maison, du côté gauche de la scène, un personnage identique de fileuse, dans le même geste que celui de Fra Angelico, pointe son regard vers l'extérieur du tableau, comme étrangère à l'évènement surnaturel qui est en train de se produire derrière le mur ${ }^{19}$. Vénus annonçant à Africo son union avec Mensola serait donc le double de l'ange qui porte la bonne nouvelle à la mère de Marie: un tel réemploi de l'iconographie de l'art sacré dans le domaine de l'art profane n'est pas insolite pour l'époque et Vittore Branca en a même signalé d'autres cas pour Boccace, mais ce qui mérite d'être souligné ici est que l'on assiste à la traduction de la fable pastorale de Boccace dans le langage pictural de Giotto, produisant ainsi la rencontre idéale entre ces deux gloires de la culture florentine ${ }^{20}$.

Sur la toile de fond de cette réception populaire de la pastorale de Boccace, particulièrement ancrée dans le patrimoine culturel de la ville et dans le sentiment de fierté communale de ses habitants, la fortune littéraire de ses ouvrages se dessine dans la continuité. Malgré un jugement mitigé porté sur Boccace par certains intellectuels humanistes comme Cristoforo Landino, la mise en valeur des auteurs du passé, véritables

19. Une reproduction de cette fresque dans Cesare GnUdi, Giotto, Milan, Aldo Martello, 1959, table XX et 74, qui décrit p. 140 l'image de la servante: "une silhouette surprenante dont le geste simple et familier atteint, dans la puissante synthèse plastique de la figure, une majesté extraordinaire». Voir aussi Giotto, La Chapelle des Scrovegni, sous la direction de G. Basile, Milan, Electa-Gallimard, 1993, p. 33. Pour la fortune iconographique de ce personnage secondaire de la fileuse on peut signaler la fresque de l'Annonciation dans l'église collégiale de San Gimignano, près de Sienne, reproduite dans le volume La Pittura in Italia. Il Duecento e il Trecento, Venise, Electa, 1986, vol. I, p. 534: cette fresque est attribuée par Cristina De Benedictis à l'entourage des Memmi. On peut la voir aussi reproduite dans Daniel ARASSE, L'Annonciation italienne. Une histoire de perspective, Paris, Hazan, 1999, p. 95, avec attribution de la fresque à Barna de Sienne ou à Lippo Menni. Daniel Arasse observe: "Barna adapte vraisemblablement, en la resserrant, la disposition inventée par Giotto pour l'Annonce à saint'Anne peinte vers 1305 à la chapelle Scrovegni de Padoue", mais d'après Raimond VAN MARLE, Iconographie de l'art profane au Moyen-Âge et à la Renaissance, La Haye, Martinus Nijoff, 1931, t. II : La vie quotidienne, p. 197, la figure de la fileuse serait plutôt un élément traditionnel dans les scènes d'Annonciation à Marie et à Sainte Anne.

20. Pour cet échange de l'art sacré à l'art profane voir Vittore BRANCA, «Il narrar boccacciano per immagini dal tardo gotico al primo Rinascimento ", in Boccaccio visualizzato, cit., vol. I, p. 327, en particulier p. 24: "tali scambi del resto, fra pittura religiosa e pittura profana, erano tutt'altro che rari in quei decenni: nei leggiadri 'deschi per parto' fiorentini è alle volte difficile distinguere le scene della nascita della Vergine o di Gesù da quelle dei pargoletti fiorentini ». 
pères fondateurs, entre dans une opération de prestige et de rayonnement, à la fois politique, culturel et linguistique, de la ville de Florence au $\mathrm{XV}^{\mathrm{e}}$ siècle: c'est à cette époque que les intellectuels florentins forgent d'ailleurs l'idéal des "Trois couronnes", promis à un grand succès au cours de la Renaissance ${ }^{21}$.

Dans ce cadre, on assiste à un véritable engouement pour le genre pastoral qui se décline selon une pluralité de registres et de styles. Le niveau le plus élevé est occupé par la tradition des églogues en latin, sans rupture de continuité par rapport aux modèles de Dante et de Pétrarque. Le thème politique y est important, en particulier sous le patronat des Médicis, grâce à l'activité de Naldo Naldi (1436-1513) qui célèbre son mécène Côme l'Ancien comme le protecteur de Florence sous le nom très symbolique d'Anthiphylos, l'ami de la fleur, à savoir de la ville de Florence (en grec philos, 'celui qui aime', et anthos, 'la fleur'). Evoquant dans la sixième églogue le retour de Côme l'Ancien à Florence après l'exil en 1434, Naldi ira jusqu'à le célébrer comme le restaurateur de l'âge d'or de Saturne ${ }^{22}$.

Plus remarquable encore est l'avènement de l'églogue en langue vernaculaire, que Leon Battista Alberti a eu peut-être le mérite d'inaugurer avec son Thyrsis, suivi à Sienne par Francesco Arzocchi et ses continuateurs, à Florence par Laurent de Médicis qui écrira en 1465 son Corinto, dont Martelli souligne l'importance: «la prima egloga volgare che fosse scritta in Firenze, e, fuori di Firenze, preceduta dal solo Tyrsis di Leon Battista Alberti ${ }^{23}$ ". Enfin, à un niveau moins engagé, on pourrait situer les allégories mythologiques d'inspiration pastorale, comme le premier livre du Quadriregio de Federico Frezzi, au début du siècle, avec sa description des

21. Lors de sa leçon inaugurale au Studium de Florence, en 1458, Cristoforo Landino affirmera: «Non m’è incognito che nella passata età Giovanni Boccaccio, uomo di grande ingegno e di non poche lettere, dette grande aiuto alla fiorentina eloquenzia, ma maggiore sarebbe stato se avessi meno perdonato alla fatica e non si fussi tanto nel dono della natura confidato che nell'arte fussi alquanto negligente; merita senza fallo grandissima laude e onore singulare, perché sempre si debbe a primi auttori delle cose portare quasi immortale reverenzia" (Cristoforo LANDINO, Scritti critici e teorici, éd. R. Cardini, Rome, Bulzoni, 1974, vol. I, p. 35).

22. M. MARTELLI, Letteratura fiorentina, cit., p. 79-86. Naldo Naldi compose ses onze églogues latines entre 1460 et 1464, une douzième en 1475. Sur les origines du pouvoir des Médicis sur Florence voir Ilaria TADDEI, "Le système politique florentin au XV siècle», in Florence et la Toscane XIV $V^{\mathrm{e}}-X I X^{\mathrm{e}}$ siècles. Les dynamiques d'un Etat italien, sous la direction de J. Boutier, S. Landi et O. Rouchon, Rennes, Presses universitaires de Rennes, 2004, p. 39-63, et la riche monographie de Dale Kent, Cosimo de' Medici and the Florentine Renaissance. The Patron's Euvre, New Haven - Londres, Yale University Press, 2000.

23. Letteratura fiorentina, cit., p. 318: "La première églogue en langue vernaculaire qui soit écrite à Florence et même hors de Florence, précédée uniquement par le Tyrsis de Leon Battista Alberti". 
royaumes de Diane et de Vénus et plus tard le Driadeo d'Amore de Luca Pulci, qui reprend de Boccace le décor pastoral et plusieurs éléments narratifs se livrant à une franche réécriture du Ninfale Fiesolano. Le succès de ces ouvrages nous informe des goûts de l'époque pour la littérature pastorale et de l'horizon d'attente du public, malgré une certaine distance culturelle par rapport au modèle de Boccace.

Luca, l'ainé des frères Pulci, est avec Luigi et Bernardo l'un des protagonistes du renouveau de la littérature en langue vernaculaire à Florence dans les dernières années de Côme de Médicis. Bernardo est l'auteur en 1460 d'une importante traduction des Bucoliques de Virgile ${ }^{24}$. Le nom de Luigi, le plus connu des frère Pulci, est associé non seulement au Morgante mais aussi à la Beca di Dicomaco, c'est-à-dire à l'évolution rustique et burlesque du genre pastoral. Luca, quant à lui, s'était tourné vers une autre source d'inspiration, se présentant comme le nouvel interprète d'Ovide dont il traduira les Heroides dans son livre de Pistole, composé vers 1466$67^{25}$. Mais ce penchant pour Ovide était déjà affiché dans le Driadeo, un poème pastoral en quatre livres qu'il avait composé quelques années plus tôt, en 1464, le dédiant au jeune Laurent de Médicis, âgé à l'époque d'à peine 15 ans $^{26}$.

Comme le Ninfale, le Driadeo est composé en huitains d'hendécasyllabes et développe une histoire tragique se terminant par la métamorphose en fleuve de deux amants, toujours dans la tradition du poème étiologique: le satyre Severe donnera son nom au fleuve Sieve et la nymphe Lora à la rivière homonyme de la région du Mugello d'où étaient originaires les

24. Marco Santagata - Stefano Carrai, La lirica di corte nell'Italia del Quattrocento, Milan, Franco Angeli, 1993, p. 105.

25. Sur les Pistole Francesca Battera, «Le Pistole di Luca Pulci e la formazione culturale del giovane Lorenzo", in Lorenzo the Magnificent Culture and Politics, cit. p. 177-190 et Donatella BisCONTI, «La Pistola I de Luca Pulci entre louange courtisane et poésie pastorale», in Chroniques Italiennes, 63-64 (2000), p. 119-138.

26. Luca Pulci, Il Driadeo d'Amore, éd. P.E. Giudici, Lanciano, Carabba, 1916. Pour un bilan sur la qualité des éditions modernes et sur la tradition manuscrite voir Paolo ROTA, «Per il Driadeo d'amore di Luca Pulci. Aspetti della tradizione manoscritta", in Studi e problemi di critica testuale, LXVIII (2004), p. 37-48. Pour la datation du Driadeo on peut se fonder sur la lettre de dédicace de Luca Pulci à Laurent de Médicis, où l'auteur rappelle le deuil public pour la mort de Côme l'Ancien, décédé le premier août 1464 à l'âge de 75 ans. Le fils de Côme, Piero il Gottoso avait alors à 46 ans la seigneurie de Florence et ses deux héritiers étaient encore très jeunes: Laurent (1449-1492) était un adolescent et son frère Giuliano (1453-78) avait tout juste 11 ans. Mais la carrière politique du jeune Laurent est précoce: en 1463 il avait déjà été envoyé comme ambassadeur en France auprès du roi Louis XI, ce qui laissait espérer un grand avenir. 
Pulci (à la Cavallina, près de Barberino du Mugello) et où les Médicis avaient leur résidence de Cafaggiolo ${ }^{27}$. Tout en souscrivant presque entièrement au jugement assez sévère de Paolo Orvieto qui définit le Driadeo un «tipico prodotto di retroguardia per l'allegorismo bucolico, per quel mitologismo d'accatto boccaccianamente eziologico e per l'occasionalità encomiastica ${ }^{28}$ ", nous nous attacherons à préciser la notion d'anachronisme, d'appartenance à une "retroguardia » culturelle: peut-on rallier, en effet, l'approche de Pulci à l'imaginaire pastoral, à celle de Boccace? Quels sont les véritables enjeux de cette réécriture du Ninfale?

Par son choix de la matière pastorale, la poésie de Luca se situe d'emblée au niveau le plus humble de la rhétorique des styles, comme l'auteur le rappelle à la fin du Driadeo: «Lauro mio, al suon de la tua cetra / da' compilar materia atta e coturna, / se questa bassa in te di grazia impetra ${ }^{29}$ ". Selon un topos virgilien connu, ici l'annonce d'un ouvrage plus engagé pourrait faire référence au livre de Pistole que Luca offrira à Laurent en 1466-1467.

Mais cette protestation d'humilité de la matière pastorale ne suffit pas à rapprocher Pulci de Boccace. Au niveau des registres expressifs, le Driadeo se caractérise en effet par son éloignement de l'élégie, de la dimension pathétique et sentimentale qui était dominante dans le Ninfale. La matière pastorale subit, chez Luca Pulci, une déformation ironique, voire burlesque, pouvant aller jusqu'à l'auto-ironie de ces vers sans doute les plus connus du Driadeo - où Luca fait allusion aux auteurs de bucoliques du Studium de Florence: "Un'accademia, uno studio di buccoici, / scandendo versi scritti da ortografi / vedrai, e sette di morali e

27. La publication de la correspondance de Laurent de Médicis est un grand projet actuellement en cours, voir Lorenzo de' Medici, Lettere I (1460-1474), éd. Riccardo Fubini, Florence, Giunti, 1977. La quatrième lettre de ce premier volume est écrite par Laurent à Cafaggiolo le 7 juin 1464, l'année de la composition du Driadeo, et contient une allusion intéressante à la fréquentation des frères Pulci. Laurent informe son père qu'il est arrivé avec Giuliano le 6 juin dans la résidence de campagne des Médicis pour fuir la contagion (de la peste?). Il ajoute qu'il a tout de suite renvoyé les frères Pulci à leur résidence de la Cavallina et qu'il a adopté avec son frère un style de vie monacal: faut-il penser que Piero craignait l'influence néfaste des Pulci sur l'éducation des jeunes princes?

28. Paolo Orvieto, «Lorenzo de' Medici e l'Umanesimo toscano del secondo Quattrocento", in Storia della Letteratuta Italiana, dir. par E. Malato, vol. 3: Il Quattrocento, Rome, Salerno Editrice, 1996, p. 295-404, p. 318.

29. Driadeo 103: «Mon cher Laurier, permet moi d'écrire / sur un sujet tragique et noble, adapté à ta lyre / si la présente matière, de genre bas, m'attire ton indulgence». 
stoici $^{30}$ ». Par ces vers qui font allusion à l'existence d'un groupe de poètes bucoliques à Florence, Pulci revendiquerait, selon Stefano Carrai, son rôle dans l'essor de la littérature pastorale: "quella autentica fioritura volgare del genere promossa appunto da lui e dai suoi fratelli ${ }^{31}$ ». Mais rien n'est dit dans le texte de la langue employée par ces poètes bucoliques, si bien que l'on pourrait penser également à des auteurs comme Naldo Naldi, si du moins l'allusion burlesque à l'orthodoxie grammaticale, les «versi scritti da ortografi», pouvait faire penser aux querelles des humanistes pour la réforme puriste du latin. Dans ce jeu parodique, en tout cas, appuyé par les rimes proparoxytones qui caractérisent le style comique, Pulci fait jouer sa veine anticonformiste, exprimant par son amusement ses distances vis-à-vis de la matière traditionnelle qu'il s'est mis à réélaborer.

Si l'on analyse le Driadeo du point de vue des motifs et des situations narratives, on retrouve naturellement plusieurs éléments du Ninfale de Boccace, étant donné l'identité de l'action principale. Ainsi, par exemple, les monologues de Severe rappellent ceux d'Africo, comme au moment de l'indécision sur la conduite à suivre devant la nymphe: sur le dilemme amour et mort se construit par exemple la plainte de Severe du huitain 50 $\mathrm{du}$ premier livre, comme déjà celle d'Africo après la première rencontre avec Mensola (Nif. Fies. 34-35).

Mais le Ninfale n'est pas un modèle exclusif, c'est tout le Boccace pastoral qui est mobilisé, réduit souvent à un style formulaire, à la simple énumération, par exemple pour la description du décor ou de ses habitants :

Comm. ninfe fior. IX 6

[Tempio] intorniato, quanto di lui si distende del vicino piano, di graziose ombre d'eccelsi pini, di diritti abeti e d'altissimi faggi e di robuste quercie.

Comm. ninfe fior. IX 7

[...] le chiare rive vi mandano le sue ninfe, e le
Driadeo I 15

Ombrava il loco altissimi cipressi lauri, faggi, folti pini e mirti e lunghi abeti $[\ldots]^{32}$.

Driadeo I 18

Napee, driope, fauni e driade,

30. Driadeo 84: «Tu verras une académie, une école de poètes bucoliques / qui déclament des vers écrits par des grammairiens forts en orthographe, / ainsi que des adeptes de la philosophie morale et stoïcienne».

31. Sefano CARraI, "Lorenzo e l'umanesimo volgare dei fratelli Pulci», in Lorenzo de' Medici. New Perspectives, Proceedings of the International Conference Held at Brooklyn College and the Graduat Center of the City University of New York, April 30-May 2, 1992, edited by Bernard Toscani, New York, Peter Lang 1994, p. 1-21, Cop. 4. 
prossime selve li fauni e le driade; e qualunque campo tiene satiri manda quivi e le naiade ancora liete vi vengono etc. satiri e ninfe il bel monte selvaggio fertile ferno $[\ldots]^{33}$.

De la Caccia di Diana aussi pourrait venir l'exploitation chez Pulci du motif de la chasse des nymphes et de la meute des chiens: une marque de ce sous-genre pastoral est représentée par l'énumération des noms des chiens de la nymphe:

Caccia di Diana. XIV 5-6

«Ciuffa! gridava "piglial buon Pezzuolo, piglial, Dragone, e piglial, Graffiacani!»

\author{
Driadeo I 73 \\ Poi gli sguinzaglia tutti i cani addosso: \\ Ripano e duo figliol parien tre bocche \\ di Cerber colla schiuma: Arion rosso \\ del seme di Lelepa e Balabocche \\ con maggior rabbia furioso e Mosso; \\ Pinamonte crudel latra e Lalocche, \\ Bonivecchio, Conzanne e Mazoricco \\ che vince i lupi, corre, e Pettoricco ${ }^{34}$.
}

Sans doute Pulci a-t-il à l'esprit également la deuxième partie du mythe d'Actéon chez Ovide, où apparaissent tous les noms des chiens de la meute d'Actéon qui vont tuer leur maître. Il s'agirait alors, dans le Driadeo, d'une reprise parodique car Severe ne succombe pas aux chiens de Lora mais il les tue sauvagement, de sorte que la nymphe pleura "come un fantin la mamma ${ }^{35}$ ».

Enfin l'influence de Boccace ne se limite pas à ses ouvrages à sujet pastoral. Cela est évident pour la séquence de la rencontre entre Severe et Lora dans la première partie du Driadeo. Il est midi; dans son vagabondage Severe se trouve devant la nymphe Lora endormie avec sa compagne Pulica et les chiens. Il la contemple, aussitôt il s'en éprend et pense qu'il

32. Comm. Ninf.: «[un temple] entouré de gracieux ombrages de très hauts pins, de sapins au tronc élancé, d'immenses hêtres et de chênes puissants, sur toute la vaste étendue de la plaine environnante»; Driadeo: «Le lieu était ombragé par de très hauts cyprès, des lauriers, des hêtres, des pins touffus, des myrtes et des sapins élancés ".

33. Comm. Ninf.: "Les claires rivières y envoient leurs nymphes et les forêts environnantes les faunes et les dryades, et toutes les campagnes habitées par des satyres les envoient ici, de même qu'y viennent les gracieuses naïades»; Driadeo: «Les napées, les dryopes, les faunes et les dryades, les satyres et les nymphes firent fécond le beau mont sauvage».

34. Chasse de Diane: "Elle criait: 'capturez-le, attrape-le mon cher Pezzuolo, attrape-le Dragone, attrape-le Graffiacani»; Driadeo: «Puis elle lâche contre lui tous ses chiens: Ripano et ses deux chiots semblaient trois gueules enragées de Cerbère: Arion le roux descendant de Lelepa et Balabocche encore plus furieux et Mosso; le cruel Pinamonte aboie avec fureur et Lalocche, Bonivecchio, Conzanne et Mazoricco, plus fort que les loups, courent de même que Pettoricco ".

35. Driadeo I 91 : «Comme une maman pleure la mort de son tout petit enfant». 
s'agit sans doute d'une déesse; il tente alors de s'approcher, mais elle se réveille et prend la fuite. Le récit du Driadeo suit évidemment l'intertexte décaméronien de la nouvelle de Cimone (Déc. V, 1), avec la différence que Boccace avait fait de Cimone un nigaud insensible par nature à l'amour, tandis que Pulci s'inspire du mythe d'Hippolyte pour présenter Severe comme un jeune orgueilleux qui a toujours refusé l'amour par dédain, comme ce sera le cas dans le Pastor Fido pour Silvio: "vantato s'era e fermo dentro al core / che nel suo petto mai regnerà Amore ${ }^{36}$ ». Le miracle ne consiste donc pas en l'éclosion de l'amour dans un esprit par nature impénétrable aux sentiments élevés, mais en la victoire de la passion sur la raison, car le renoncement orgueilleux de Severe à l'amour va se transformer en une passion aveugle qui le conduira à la mort. Fidèle au decorum tragique de son personnage, Pulci lui réserve des monologues plaintifs et un rôle plutôt passif dans l'histoire, sans jamais le représenter en train d'échafauder le viol de la nymphe, comme Africo dans le Ninfale, ce qui témoigne d'une intention de censure comparable à la version épurée que le peintre Giovanni Toscani avait peinte sur son coffre à une époque assez proche.

Force est de constater, alors, que Pulci ne cherche pas à se démarquer de Boccace au niveau des thèmes et des situations narratives. En revanche, il affiche une grande liberté au regard de la structure du poème: il oppose, en effet, à la linéarité du récit du Ninfale, qui était axé sur le développement d'une seule action dramatique, une logique narrative émiettée et centrifuge, par emboîtement de récits dans les récits, de façon à retarder le dénouement de l'action. Non seulement il se passe trois ans entre la rencontre des amants et leur fin tragique, mais le récit de leur aventure est entrecoupé par nombre d'épisodes secondaires qui rappellent vaguement la technique des cantari. Ce choix est intentionnel: Pulci s'inspire de la structure narrative des Métamorphoses d'Ovide et de ce fait semble vouloir corriger Boccace par son archétype classique.

La plupart de ces divagations sont constituées par des récits mythiques et en ce sens Pulci s'attache à rendre explicites et à développer les motifs ovidiens que Boccace avait laissé implicites. La seule allusion explicite de Boccace à Ovide apparaît vers la fin du Ninfale, lorsque Mensola découvre sa grossesse. Pulci en revanche exhibe ce modèle dès la première apparition de Lora, tout en rajoutant le mythe de Cyparissus:

36. «Il avait décidé et s'en était fait fort / que jamais Amour ne serait maître de son coeur ». 
Ninfale 333

I' posso esser annoverata omai, o Caliston, con teco, che com'io già fosti ninfa, e poi con molti guai Diana ti cacciò per ogni rio, perché Giove t'ingannò, come sai, ed in orsa, crudel, ti convertìo; e givi errando, e le cacce temevi, mugghiando quando favellar volevi.
Driadeo IV 45

Se il suon del corno ti rimbomba presso conoscer puoi ch'io non Calisto sia che abbia il ventre di lussuria oppresso; né per selve, temeraria e ria ho morto il cervo come fé Cipresso; ma son la Lora tua nipote e pia la qual, qui fredda, a quest'ombra m'assidero e far la voglia tua sempre desidero ${ }^{37}$.

Si Boccace avait dissimulé ses emprunts à Ovide, c'était au profit d'une dynamique naturaliste de son récit, que tous les interprètes ont loué pour sa linéarité de fable populaire ${ }^{38}$, alors que Pulci met en exergue l'ascendance érudite de son jeu littéraire.

Un autre passage du Driadeo exploitant longuement un motif ovidien occupe plusieurs huitains du troisième livre: le fleuve Tavaiano cherchant à empêcher le suicide de Severe lui raconte d'abord l'histoire de son amour heureux pour la nymphe Estura et enchaîne ensuite avec une ancienne histoire d'amour: "Poi cominciai d'amor commedia vetera" (Driadeo 53). Le mythe d'Atalante et Hippomène des Métamorphoses d'Ovide est repris comme dans un exercice de traduction littérale allant jusqu'au mot à mot:

\section{Ovide, Métam. X} v. 560-561

Forsitan audieris aliquam certamine cursus veloces superasse viros

v. $571-72$

Praemia veloci coniunx thalamique dabuntur mors pretium tardis: ea lex certaminis esto

v. 661-62

O quotiens, cum iam posset transire, morata est

Spectatosque diu vultus invita reliquit

\section{Driadeo 53}

Forse sentito arai come Atalanta

Vergine greca gloriosa e bella

Correr pedestra più che alcun si vanta

55

[...] chi mi vince m'abbi in prezzo, e morte in guiderdon riceva il sezzo.

69

E potendo ella il giovine passare più volte nel suo corso si rattenne tanta pietà del suo amador le venne.

37. Ninf. Fies. : «Désormais, o Callisto, ma légende sera comparable à la tienne, toi qui fus jadis une nymphe et qui déversas tant de larmes, chassée de partout par Diane car Jupiter t'avait séduite par la ruse, comme tu le sais, et la déesse cruelle te changea en ourse: tu t'en allais fugitive, en craignant les chasses et en beuglant quand tu voulais parler»; Driadeo: "Si le son du cor résonne jusqu'à toi, tu peux savoir que je ne suis pas Callisto, grosse à cause de sa luxure et que je n'ai pas tué le cerf comme le fit Cyparissus parcourant les forêts, mais je suis la Lore, ta nièce dévote et sous cet ombrage transie et engourdie de froid, je ne désire que de suivre toujours ta volonté».

38. Voir par exemple les remarques d'Armando Balduino: "una narrazione che nulla concede, poi, nel suo nitido e compatto sviluppo, a divagazioni erudite o a dotti indugi, dissonanti con la sua popolaresca linearità», Ninf. Fies., éd. cit., p. 277. 
v. 679

neve meus sermo cursu sit tardior ipso
73

Ed acciò ch'io non faccia il mio sermone più lungo che il corso di costoro 39

Dans ce cas c'est le texte du Driadeo qui nous invite à reconsidérer l'élément ovidien dissimulé chez Boccace: le passage du Ninfale 99-115 où Mensola fuit son amant dans la brousse, découvrant «le gambe e 'l ginocchio vezzoso / ch'ognun ne diverria desideroso" et inspirant chez Africo un mouvement de compassion ( «i priego Giove che' I monte e la costa / ispiani tutta [...] / e pianura diventi umile e piana», 106), pourrait être rapproché de la fuite de Daphné poursuivie par Apollon (motif si cher à Pétrarque), mais dans la deuxième partie, lorsque les sentiments de Mensola évoluent de la peur à la pitié, c'est bien le mythe d'Atalante qui apparaît en filigrane:

Quand'ella il dardo per l'aria vedea

zufolando volar, e poi nel viso

guardò del suo amante, il qual parea

veracemente fatto in Paradiso,

di quel lanciar forte se ne pentea,

e tocca di pietà lo mirò fiso,

e gridò forte - Omè, giovane, guarti,

ch'i'non potrei omai di questo atarti! 40

De même Atalante est partagée, à la vue du jeune Hippomène, entre son désir de remporter la victoire et l'instinct de sauver son adversaire dont la mort serait pour elle trop cruelle, car elle l'aime déjà sans pouvoir s'en douter (Métam. X 637: «amat et non sentit amorem»).

39. Ovide, dans la trad. de G. Lafaye (Les Métamorphoses, Paris, Les Belles Lettres, 1928), v. 560-561: «Il y avait une femme, peut-être en as-tu entendu parler, qui surpassait à la course même des hommes agiles»; v. 571-572: «Le plus agile recevra pour prix de sa victoire ma main et mon lit; ceux que j’aurais devancés paieront de la vie leur défaite»; v. 661-662: «oh! que de fois, pouvant passer la première, elle s'est attardée à regarder le cher visage et ne l'a quitté des yeux qu'à regret"; v. 679: "enfin, pour que mon récit ne soit pas encore plus lent que sa course elle-même». Driadeo 53: "On t'a déjà sans doute raconté d'Atalante / vierge grecque pleine de gloire et de beauté / qui se vante d'être la première au monde dans la course à pied "; $55:$ «je serai le prix pour celui qui gagne / et le dernier aura en gage la mort»; 69: "bien que capable de doubler le jeune homme, / à plusieurs reprises elle ralentit sa course / pour la grande compassion qui la prit envers son amant»; 73: "et afin que mon récit ne dure pas / plus longtemps que la course de ceux-ci».

40. "Lorsqu'elle vit son propre dard s'envoler dans les airs et regarda ensuite le visage de son amant, qui semblait vraiment fait au Paradis, se repentit aussitôt de l'avoir décoché et touchée de compassion le regarda bien droit et cria fort : - Hélas, jeune homme, prends garde à toi, je ne pourrais désormais te défendre de ceci». 
En conclusion, la poésie pastorale de Luca Pulci propose une redéfinition des codes traditionnels tout en suivant le schéma du poème mythologique et pastoral. Les emprunts à Boccace suivent une logique de la contamination, puisant aussi bien dans ses ouvrages à sujet pastoral que dans le Décaméron, et l'élément érudit et savant est exhibé jusqu'à la caricature. Il est alors possible de reconnaître dans le Driadeo une double pédagogie, à la fois littéraire et amoureuse, à l'intention du jeune Laurent, le dédicataire du livre: par les détours d'une écriture plaisante et enjouée, le prince est appelé à faire son apprentissage d'Ovide tout en apprenant, lui qui en 1464 avait déclaré sa flamme à la belle Lucrèce Donati, comment les affres de l'amour sont matière à rire et à pleurer, mais dans le domaine de la littérature.

La poésie de Pulci se veut donc un langage d'initiés, se laisse lire au deuxième degré par un renvoi incessant à la tradition littéraire. Elle se caractérise enfin, s'éloignant encore une fois de l'esprit du Ninfale, par son intention panégyrique déclarée, comme le montre l'épisode central de la joute entre Tavaiano et le personnage de Lauro, le double pastoral de Laurent de Médicis. Au moment où le vieux Tavaiano a réussi à attirer la nymphe Estura près d'une rivière dans l'intention de la séduire, un jeune homme à cheval arrive sur les lieux accompagné de son frère cadet et offre sa protection à la nymphe: il s'agit de Lauro, fils d'Apollon. Une compétition s'engage alors entre Lauro et Tavaiano pour établir qui des deux sera l'élu de la belle, l'occasion de faire prononcer au prince l'éloge de la ville de Florence "una città regina / d'altre città " (Driadeo III 79). Une trame d'allusions à la chronique contemporaine, à des faits et des personnages de l'actualité, vient interrompre la fiction pastorale, ce que Boccace ne s'était pas autorisé. On reconnaît par exemple la figure de Côme derrière l'éloge que Lauro fait de son aïeul:

Poi altamente il dolce verso muove

all'avol degno che suo petto spiri

il qual per grazia siede in grembo a Giove,

quello del mondo e de' celesti giri

cosmografo perfetto e specchio dove

chi vuol trovar virtù convien che miri,

padre alla patria e fondator di pace

e del tempio nel qual si cuba e giace ${ }^{41}$.

41. Driadeo III 21 : «Ensuite il commence à chanter doucement / priant son noble aïeul d'inspirer sa poésie, / celui qui est bienheureux dans le giron de Jupiter / et cosmographe expert du monde et des sphères célestes, / miroir de toutes vertus pour qui voudrait les apprendre, / père de la patrie et fondateur de la paix et du temple où l'on donne sépulture». 
Le deuil public pour la mort de Côme en 1464 est rappelé dans l'épître à Laurent de Médicis en ouverture du Driadeo. Ici, Pulci prononce par voie indirecte son oraison funèbre, rappelant que l'ancien maitre de la ville a mérité le Paradis, et qu'il s'était illustré non seulement par ses vertus mais aussi par sa dévotion à la culture et à la politique. La qualification de "cosmografo perfetto", renvoyant par un jeu étymologique au nom 'Cosmo', variante de 'Cosimo' (du grec cosmos: 'bien ordonné), fait référence aux célèbres intérêts de Côme pour la géographie et l'astrologie qui avaient permis d'importantes avancées scientifiques à Florence, grâce à la diffusion des ouvrages de Ptolémée et de Strabon, à leur traduction et aux recherches menées par Paolo Toscanelli et Gemiste Pléton ${ }^{42}$. La louange courtisane de Luca Pulci rejoint en outre le témoignage du libraire Vespasiano de Bisticci quant à la passion de Côme pour l'astrologie: «se erano astrolagi, egli n'aveva uno universale giudicio, per avere sempre praticato con maestro Pagolo e con altri astrolagi; in qualche cosa vi dava fede e usavala in alcuna sua $\operatorname{cosa}^{43} »$. Ensuite, Pulci célèbre le grand-père de Laurent comme "padre alla patria e fondator di pace» en réemployant l'épithète de pater patriae qui fut conféré à Côme lors de ses funérailles publiques $^{44}$. C'est le Côme-Saturne que Naldo Naldi avait déjà transfiguré dans sa sixième églogue, signe d'un fond idéologique et politique commun ralliant Pulci au «studio di buccoici» de la Florence des Médicis. Enfin, une dernière remarque concerne l'allusion au temple où «si cuba e giace» que Côme aurait fondé: protecteur des arts et de l'architecture, mécène d'artistes comme Brunelleschi, Michelozzo, Benozzo Gozzoli e Fra Angelico, Côme l'Ancien avait financé d'importants travaux d'embellissement de la ville de Florence, comme la construction du couvent dominicain de Saint Marc ou de la basilique de Saint Laurent, où se trouvent les tombeaux de la famille Médicis.

42. D. Kent, Cosimo de' Medici, cit., p. 90. Grâce à Côme on assiste à Florence à un grand développement de la cartographie: «by the early years of the sixteenth the Medici were the owners of one of the finest collections of maps in Europe" (ibid., p. 264).

43. Vespasiano DA Bisticci, Vite di uomini illustri del secolo $X V$, éd. P. D'Ancona et E. Aeschlimann, Milan, Hoepli, 1951, p. 418: «quand il parlait avec des astrologues, il s'y connaissait très bien car il avait toujours fréquenté messire Paolo [Toscanelli] et d'autres. Il tenait en considération l'astrologie pour certains aspects et il s'en servait en certaines occasions".

44. Ce titre élogieux était utilisé pour Côme même avant sa mort, par exemple par Feo Belcari, cf. D. Kent, Cosimo de' Medici, cit., p. 18. 
Cherchant à définir l'héritage de Boccace pastoral à l'époque des frères Pulci, nous nous sommes confrontés non pas à une arrière-garde culturelle - ce qui impliquerait un jugement négatif vis-à-vis de Boccace, comme d'un auteur devenu inactuel pour le public de la Renaissance mais plutôt à une opération complexe et idéologiquement orientée, capable de développer les aspects novateurs du magistère de Boccace: la reprise des mythes ovidiens, le rattachement de la matière pastorale aux origines de la ville de Florence, l'organisation du discours autour d'une histoire d'amour tragique sont autant d'éléments de continuité que Pulci a su réinterpréter tout en enrichissant son poème pastoral de nouveaux éléments, comme l'intention panégyrique qui semble annoncer la poésie courtisane, le jeu de masques renvoyant à l'actualité, la veine parodique et la dérive comique du style humble de tradition bucolique (l'emploi des rimes proparoxytones en est la preuve la plus évidente). Loin de se résumer à un simple jeu d'érudition littéraire, le Driadeo laisse apparaître, d'autre part, une réflexion sur les enjeux de la poésie pastorale qui pourrait nous éclairer sur un aspect problématique du Ninfale. Nous revenons ainsi à la scène du viol et à sa nécessité narrative: faudrait-il l'imputer à une certaine misogynie de fond chez Boccace?

Dans certains passages du Driadeo, Pulci développe le thème de l'opposition entre la loi du plaisir, propre aux dieux de l'antiquité, et les lois imposées par la société: au premier livre, par exemple, une nymphe amoureuse de Severe et rejetée par celui-ci s'en prend aux divinités en criant: «Lecito è fare a voi quel che vi piace / e chi con ninfe e chi con altri giace ${ }^{45}$ ». Un esprit de fronde qui réapparaît dans la partie finale du poème, au quatrième livre, au moment où Severe fait le triste bilan de ses années de fidélité infructueuse: «Era passato l'anno terzo e 'l mese / che 'l bel satiro Severe nel bosco / per la driada Amor di fiamma accese ${ }^{46}$ ». La déesse des enfers, Proserpine, envoie alors à son secours une horrible sorcière, Urade, qui se met à préparer une nouvelle rencontre du satyre avec la nymphe. En attendant la venue de Lora, Severe s'abandonne à son rêve d'amour imaginant que la loi de chasteté imposée par Diane soit définitivement révoquée:

Che frutto è ne' mortali seguir Diana

ed andar drieto sempre a fiere e boschi?

45. Driadeo I 39: «Il vous est permis de faire ce qui vous plait, de vous accoupler avec les nymphes ou autres partenaires".

46. Driadeo IV 1 : «Trois ans et un mois étaient passés / depuis que le beau satyre Severe dans le bois, / victime d'Amour s'était épris de la dryade». 
Spengasi or le leggi di Diana

e' boschi, e seguitiam tutti l'Amore:

Diana e Vesta sien lungi del core ${ }^{47}$.

Aussi, l'exemple de l'infidélité de Jupiter lui dicte-t-il une nouvelle loi fondée sur la légitimité du plaisir :

Per esemplo divin vediam che Giove
di Saturno le leggi dà allo inferno
e fra le ninfe fa d'amor gran prove
e 'l nome suo è sopra gli altri eterno;
e Giunon che le nubi in aria move
gli è sora e moglie e tale esemplo dierno,
al mondo alluminando nostre legge
che lecito è ciò che desio elegge ${ }^{48}$.

Bien avant l'Aminta et le Pastor Fido, ce motif de l'interdit bravé, du retour à une conception naturelle de l'amour, réélaboré ici à partir des célèbres vers de Dante sur la luxure de Sémiramis «che libito fé licito in sua legge" (Enfer V, 56), permet de donner libre cours à des thèmes comme la transgression, le désir, le conflit entre nature et culture ${ }^{49}$. Pulci avait-il pu lire cette problématique dans le Ninfale? Certes, l'action dramatique chez Boccace s'enchaîne à partir du motif de l'interdit: la violation de la loi de chasteté imposée par Diane rend Mensola impie et digne de la mort. Cependant, Boccace ne va pas jusqu'à souligner l'opposition entre le devoir et le désir, c'est plutôt la mort des amants qui suffit à elle seule à sanctionner la transgression. D'autre part, aucun brouillage n'est possible, dans le Ninfale, entre fiction et réalité, entre temps du mythe et présent de l'histoire, car la mésaventure d'Africo et Mensola appartient à un passé révolu et toute la deuxième partie du poème se concentre sur le destin de Pruneo, le fils de Mensola, et sur sa descendance qui assurera la continuité entre Fiesole et Florence. Dans l'espace-temps circonscrit de cette préhistoire de l'humanité, Boccace a sans doute prêté à Africo les traits d'un héros des mythes ovidiens, cette sensualité insouciante et instinctive d'un Jupiter pour qui, comme l'imaginera Pulci, «lecito è ciò che desio elegge».

47. Driadeo IV 54: «Qu'ont il à gagner les mortels en suivant Diane / et en se vouant toujours aux fauves et aux forêts? / Assez à présent des lois de Diane / et des forêts, suivons tous l'Amour / que Diane et Vesta soient loin de nos cœurs".

48. Driadeo IV 55: «Prenant exemple d'un dieu nous voyons que Jupiter / se moque grandement des lois de Saturne / et se livre parmi les nymphes à ses exploits d'amour / et son nom reste éternel au dessus de tous / et Junon qui commande aux nuages / est sa sœur et son épouse, et ils nous offrirent leur modèle, / répandant par le monde nos lois: / 'licite est ce que le désir choisit'”.

49. "S'ei piace, ei lice» chante le chœur de l'acte I de l'Aminta, auquel Guarini oppose la loi de l'honneur, "piaccia, se lice», dans le chœur de l'Acte IV du Pastor Fido. 